\title{
Modal Interpretations of Quantum Mechanics and Relativity: A Reconsideration
}

\author{
Joseph Berkovitz* Meir Hemmo ${ }^{\dagger}$
}

Two of the main interpretative problems in quantum mechanics are the socalled measurement problem and the question of the compatibility of quantum mechanics with relativity theory. Modal interpretations of quantum mechanics were designed to solve both of these problems. They are no-collapse (typically) indeterministic interpretations of quantum mechanics that supplement the orthodox state description of physical systems by a set of possessed properties that is supposed to be rich enough to account for the classical-like

* Department of Philosophy, University of Maryland Baltimore County, 1000 Hilltop Circle, Baltimore, MD 21250, USA; Email: jberkov@umbc.edu

$\dagger$ Department of Philosophy, University of Haifa, Haifa 31905, Israel; Email: meir@research.haifa.ac.il 
behavior of macroscopic systems, but sufficiently restricted so as to avoid the no-hidden-variables theorems. But, as recent no-go theorems suggest, current modal interpretations are incompatible with relativity. In this paper, we suggest a strategy for circumventing these theorems. We then show how this strategy could naturally be integrated in a relational version of the modal interpretation, where quantum-mechanical states assign relational rather than intrinsic properties.

KEY WORDS: modal interpretations, relativity, Lorentz invariance, relational modal interpretation, relational properties.

\section{INTRODUCTION}

The measurement problem arises in orthodox, no-collapse quantum mechanics from two features that account very successfully for the behavior of microscopic systems: The linear dynamics of quantum states (the Schrödinger equation in the non-relativistic case) and the so-called 'eigenstate-eigenvalue link.' Microscopic systems may be in superposition states of position, mo- 
mentum, energy, as well as various other physical observables. According to the eigenstate-eigenvalue link, an observable of a system has a definite value (one of its eigenvalues) just in case the system is in the corresponding eigenstate of that observable. Accordingly, microscopic systems may be in a state of indefinite position, momentum, energy or other physical quantities. The measurement problem is that given the linear and unitary Schrödinger dynamics, these indefinite quantities are also endemic in the macroscopic realm. For example, during a $z$-spin measurement on a particle in a superposition state of z-spin 'up' and z-spin 'down' the position of the apparatus' pointer gets entangled with the indefinite $z$-spin of the particle, thus transforming the pointer into a state of indefinite position, i.e. a superposition of pointing 'up' and pointing 'down.' Since this indefiniteness is generic in orthodox no-collapse quantum mechanics, measurements typically have no definite outcomes.

The no-collapse 'hidden-variables' interpretations of quantum mechanics provide a major strategy for addressing the measurement problem. They postulate that the quantum-mechanical state description of systems is incomplete and supplement it with rules for assigning additional definite properties 
over and above the ones picked out by the eigenstate-eigenvalue link. And while the dynamics of quantum states of isolated systems is assumed to obey linear and unitary equations of motion (the Schrödinger equation in the nonrelativistic case), and accordingly quantum states never 'collapse' systems in superposition of properties may sometimes possess one of these properties. Thus, for example, a pointer in a superposition of pointing to 'up' and pointing to 'down' may be in a definite state of pointing to either 'up' or 'down.' More generally, the idea is that the set of the additional definite properties will be rich enough to account for the occurrence of definite macroscopic events, including measurement outcomes, but sufficiently restricted so as to avoid the no-hidden-variables theorems; and the dynamics of these properties will reproduce the familiar classical-like behavior of macroscopic systems.

While various no-collapse hidden-variables interpretations of quantum mechanics seem to provide a more or less satisfactory solution to the measurement problem in the non-relativistic realm, the challenge is to show that such theories could be compatible with relativity theory. In particular, the challenge is to show that in such theories the dynamics of properties is fundamentally Lorentz invariant, i.e. satisfies the Lorentz transformations without 
picking out any reference frame as preferred.

Modal interpretations of quantum mechanics were designed to solve the measurement problem and to reconcile quantum mechanics with relativity. They are no-collapse (typically) indeterministic interpretations that supplement the orthodox quantum-mechanical state description of systems by a set of properties that is supposed to be rich enough to account for the occurrence of definite macroscopic events and their classical-like behavior, but sufficiently restricted to escape all the known no-hidden-variables theorems. (For reviews and analysis of modal interpretations, see Dieks and Vermaas ${ }^{(1)}$ and Dickson ${ }^{(2)}$, and references therein. For a brief review of the main property assignments in current modal interpretations, see Sec. 2.)

But, current modal interpretations face two major problems. First, although intended to solve the measurement problem, the mainstream modal interpretations (which only assign properties in the Schmidt bases) fail to explain how classical-like behavior of macroscopic systems arises from their property assignment. In particular, it turns out that in certain situations of environmentally induced decoherence, the property assignment of these 
interpretations does not pick out the familiar classical-like properties. ${ }^{(3)}$ Second, no-go theorems by Dickson and Clifton ${ }^{(4)}$, Arntzenius ${ }^{(5)}$ and Myrvold ${ }^{(6)}$ suggest that all current modal interpretations are not genuinely relativistic. Dickson and Clifton ${ }^{(4)}$ demonstrate that if modal interpretations are to satisfy a certain stability condition, namely the assumption that the possessed properties of a 'freely' evolving system follow its unitary evolution, and some other natural assumptions about the evolution of properties and their probabilities, then the transition probabilities of future possessed properties of a system given its current possessed properties will sometimes fail to be genuinely Lorentz invariant. Arntzenius ${ }^{(5)}$ argues that the stability assumption is dispensable and the core of Dickson and Clifton's no-go theorem concerns the non-existence of a joint distribution yielding the single-time Born-like probabilities for possessed properties as marginals. ${ }^{1}$ And Myrvold ${ }^{(6)}$ provides a generalization of Arntzenius's version of Dickson and Clifton's no-go theorem. Given these theorems, the common view is that modal interpreta-

\footnotetext{
${ }^{1}$ Here and henceforth, by "single-time Born-like probabilities" or in short "Born probabilities," we shall always mean single-time probabilities of properties (i.e. probabilities that systems have properties at a certain time or state), computed according to a Bornlike rule (for more details, see Sec. 2 and 5.1). This is a generalization of the domain of application of the Born rule from measured properties to all possessed properties.
} 
tions of quantum mechanics are not genuinely relativistic.

In this paper, we consider the question of the compatibility of modal interpretations with special relativity, focusing on Myrvold's no-go theorem. We suggest a strategy for circumventing this theorem, and then show how it could naturally be integrated in a relational version of the modal interpretation. In contrast to the mainstream modal interpretations, in which the core-property assignment is of intrinsic properties, in this interpretation quantum states assign only relational properties. In Sec. 2, we briefly present the core-property assignment of the current modal interpretations, and in Sec. 3 we review Myrvold's no-go theorem. In Sec. 4, we propose a strategy for circumventing Myrvold's theorem. In Sec. 5, we show how this strategy can be integrated in a relational modal interpretation; ${ }^{2}$ an in-

\footnotetext{
${ }^{2}$ For other relational versions of the modal interpretation, see the perspectivalist modal interpretations by Kochen ${ }^{(7)}$ and Bene and Dieks ${ }^{(8)}$. We believe that these versions of the modal interpretation are also subjected to Myrvold's no-go theorem, but for want of space we are unable to discuss them in this paper. For other non-modal relational interpretations of quantum mechanics, see Everett ${ }^{(9)}$, Rovelli ${ }^{(10,11)}$ and Saunders ${ }^{(12,13)}$. Again, for want of space, we are unable to discuss the prospects of these interpretations with respect to
} 
terpretation that also circumvents Dickson and Clifton's and Arntzenius's theorems. Our strategy postulates that the values of physical quantities that are commonly thought of as local, such as pointer positions, are not really local. In Sec. 6 we argue that this type of nonlocality is unobservable and, moreover, we explain how in spite of the relational nature of properties our experience could be accounted for.

\section{THE PROPERTY ASSIGNMENTS IN CURRENT MODAL INTERPRETATIONS}

Modal interpretations vary in their property assignment. There are three main types of property assignments. In some modal interpretations, the property assignment is based on the Schmidt biorthogonal-decomposition

theorem. ${ }^{(15-17)}$ Let $S_{1}$ and $S_{2}$ be systems associated with the Hilbert spaces $\mathcal{H}^{S_{1}}$ and $\mathcal{H}^{S_{2}}$ respectively, and let

$$
|\psi\rangle_{S_{1}+S_{2}}=\sum_{i} c_{i}\left|\alpha_{i}\right\rangle_{S_{1}}\left|\beta_{i}\right\rangle_{S_{2}}
$$

be the unique biorthogonal decomposition of the (quantum-mechanical) state Myrvold's theorem. For a short review of the relational quantum theories mentioned above, see Laudisa and Rovelli ${ }^{(14)}$. 
of $S_{1}+S_{2}$. Then, $S_{1}$ has a determinate value for each $\mathcal{H}^{S_{1}}$ observable with eigenbasis $\left\{\left|\alpha_{i}\right\rangle\right\}$ and $S_{2}$ has a determinate value for each $\mathcal{H}^{S_{2}}$ observable with eigenbasis $\left\{\left|\beta_{i}\right\rangle\right\}$, and $\left|c_{i}\right|^{2}$ give the single-time Born-like probabilities for the possible values of these observables, which are typically interpreted as long-run frequencies of these values. If some of the $c_{i}$ are degenerate, the Schmidt biorthogonal decomposition is not unique, and the properties assigned by the above rule are projections onto multi-dimensional subspaces.

In other modal interpretations, such as Vermaas and Dieks ${ }^{(18)}$, the property assignment for a system is based on its reduced state (obtained by partial tracing). Let the reduced state of a system $S$ be $\rho$, and let the unique spectral resolution of $\rho$ be

$$
\rho=\sum_{i}\left|\lambda_{i}\right|^{2} P_{i}
$$

where $P_{i}$ are the eigenprojections of $\rho$. Then, the single-time Born-like probability that $S$ possesses a property represented by $P_{i}$ is $\left|\lambda_{i}\right|^{2} \cdot \operatorname{dim}\left(P_{i}\right)$. This property assignment is (in a sense) a generalization of the Schmidtdecomposition based property-assignment, as it could also be applied to mixed states. 
In both the Schmidt-decomposition and the spectral-resolution modal interpretations, the set of definite physical quantities, i.e. observables with definite values, generally varies over time. By contrast, in Bub's modal interpretation $^{(19)}$ the definite quantities of a system are always given by the non-zero projections of the system's (quantum-mechanical) state onto the eigenvectors of some particular preferred observables ${ }^{3}$ (typically macroscopic observables). These preferred observables are distinguished from other observables in that their behavior is stable under environmentally induced decoherence.

The above property assignments may apply to any partition of the universe, or more precisely to any factorization of the Hilbert space of the universe. But in some modal interpretations, the so-called atomic modal interpretations ${ }^{(21,22)}$, the core-property assignment is confined to a preferred factorization; the factorization of the Hilbert space of the universe into the Hilbert spaces of its atomic subsystems. And the properties of (composite) systems are inherited from the properties of their atomic subsystems.

\footnotetext{
${ }^{3}$ In this sense, Bub's modal interpretation is similar to Bohm's pilot-wave theory ${ }^{(20)}$, which stipulates that the position of systems in configuration space is always determinate.
} 
Though these versions of the modal interpretations are also subjected to the existing no-go theorems for relativistic modal interpretations, in what follows we shall focus on the non-atomic modal interpretations.

\section{MYRVOLD'S NO-GO THEOREM FOR RELATIVISTIC MODAL INTERPRETATIONS}

Myrvold argues that all the property assignments above are incompatible with special relativity, independently of their dynamics for possessed properties. ${ }^{(6)}$ The main idea of the argument is the following. Myrvold presupposes that if a modal interpretation is to be compatible with relativity, it must satisfy the following condition (Ref. 6, p. 1783):

Relativistic Born Rule. Let $q$ and $r$ be any possible values of the observables $Q_{1}$ and $R_{2}$ respectively, and suppose that $Q_{1}=q$ and $R_{2}=r$ are local definite properties of the systems $S_{1}$ and $S_{2}$ respectively. For any spacelike hypersurface $\alpha$, if the quantum-mechanical state of the composite system $S_{1}+S_{2}$ on $\alpha$ is $\psi(\alpha)$, then the probability of $Q_{1}=q$ 
and $R_{2}=r$ on $\alpha$ is equal to $\operatorname{Tr}\left[P_{Q_{1}}(q) P_{R_{2}}(r) \psi(\alpha)\right]$; where $P_{Q_{1}}(q)$ and $P_{R_{2}}(r)$ are the projections onto the eigenspaces $Q_{1}=q$ and $R_{2}=r$, respectively.

INSERT FIGURE 1 ABOUT HERE (attached in a separate file)

But, argues Myrvold, this condition is incompatible with all the above property assignments. His reasoning is the following. Let $\alpha$ and $\beta$ be two hyperplanes of simultaneity in some reference frame (see Fig. 1 above). Let $x_{i}$ be a small region on $\alpha$ in which $S_{i}$ is located, and let $y_{i}$ be a small region on $\beta$ in which $S_{i}$ is located. Suppose that $x_{1}$ is spacelike separated from $y_{2}$ and $x_{2}$ is spacelike separated from $y_{1}$. Let $\gamma$ be a spacelike hypersurface containing $y_{1}$ and $x_{2}$ and let $\delta$ be a spacelike hypersurface containing $x_{1}$ and $y_{2}$. Let $R_{1}$ and $R_{2}$, associated with the systems $S_{1}$ and $S_{2}$ respectively, be observables that have definite values on $\alpha, \beta, \gamma$ and $\delta$ according to all the above property assignments. This will be the case if $R_{1}$ and $R_{2}$ are observables that have definite values in Bub's modal interpretation, and $S_{1}$ and $S_{2}$ are coupled with measuring devices $A_{1}$ and $A_{2}$, which record the values of $R_{1}$ and $R_{2}$ on $\alpha, \beta$, 
$\gamma$ and $\delta$, so that the states of $S_{1}+S_{2}+A_{1}+A_{2}$ on these hypersurfaces are:

$$
\begin{aligned}
|\varphi(\alpha)\rangle= & 1 / 2 \sqrt{3}\left(\left|p_{1}+\right\rangle\left|r_{1}+\right\rangle\left|r_{2}+\right\rangle\left|p_{2}+\right\rangle-\left|p_{1}+\right\rangle\left|r_{1}+\right\rangle\left|r_{2}-\right\rangle\left|p_{2}-\right\rangle-\right. \\
& \left.-\left|p_{1}-\right\rangle\left|r_{1}-\right\rangle\left|r_{2}+\right\rangle\left|p_{2}+\right\rangle-3\left|p_{1}-\right\rangle\left|r_{1}-\right\rangle\left|r_{2}-\right\rangle\left|p_{2}-\right\rangle\right) \\
|\varphi(\beta)\rangle= & 1 / \sqrt{3}\left(\left|p_{1}+\right\rangle\left|r_{1}+\right\rangle\left|r_{2}-\right\rangle\left|p_{2}-\right\rangle+\left|p_{1}-\right\rangle\left|r_{1}-\right\rangle\left|r_{2}+\right\rangle\left|p_{2}+\right\rangle-\right. \\
& \left.-\left|p_{1}+\right\rangle\left|r_{1}+\right\rangle\left|r_{2}+\right\rangle\left|p_{2}+\right\rangle\right) ; \\
|\varphi(\gamma)\rangle= & 1 / \sqrt{6}\left(\left|p_{1}-\right\rangle\left|r_{1}-\right\rangle\left|r_{2}+\right\rangle\left|p_{2}+\right\rangle+\left|p_{1}-\right\rangle\left|r_{1}-\right\rangle\left|r_{2}-\right\rangle\left|p_{2}-\right\rangle-\right. \\
& \left.-2\left|p_{1}+\right\rangle\left|r_{1}+\right\rangle\left|r_{2}-\right\rangle\left|p_{2}-\right\rangle\right) \\
& \\
& \\
& \left.-2\left|p_{1}-\right\rangle\left|r_{1}-\right\rangle\left|r_{2}+\right\rangle\left|p_{2}+\right\rangle\right) ; \\
|\varphi(\delta)\rangle= &
\end{aligned}
$$

where for each $i,\left|r_{i}+\right\rangle$ and $\left|r_{i}-\right\rangle$ are distinct eigenstates of the observable $R_{i}$, and $\left|p_{i}+\right\rangle$ and $\left|p_{i}-\right\rangle$ are distinct eigenstates of the pointer observable $P_{i}$, associated with the measuring device $A_{i}$. As is easily seen, $|\varphi(\beta)\rangle,|\varphi(\gamma)\rangle$ and $|\varphi(\delta)\rangle$ are obtained from $|\varphi(\alpha)\rangle$ by applying the following Hadamard transformations to the eigenstates of $R_{i} \otimes P_{i}$ :

$$
\begin{aligned}
& U_{i}\left|r_{i}+\right\rangle\left|p_{i}+\right\rangle=1 / \sqrt{2}\left(\left|p_{i}+\right\rangle\left|r_{i}+\right\rangle+\left|p_{i}-\right\rangle\left|r_{i}-\right\rangle\right) \\
& U_{i}\left|r_{i}-\right\rangle\left|p_{i}-\right\rangle=1 / \sqrt{2}\left(\left|p_{i}+\right\rangle\left|r_{i}+\right\rangle-\left|p_{i}-\right\rangle\left|r_{i}-\right\rangle\right) .
\end{aligned}
$$


That is, $|\varphi(\beta)\rangle=U_{1} \otimes U_{2}|\varphi(\alpha)\rangle,|\varphi(\gamma)\rangle=U_{1} \otimes I_{2}|\varphi(\alpha)\rangle$ and $|\varphi(\delta)\rangle=$ $I_{1} \otimes U_{2}|\varphi(\alpha)\rangle$; where $I$ is the identity transformation.

Suppose further that the possession of a definite value of $R_{i}$ is a local property of the system $S_{i}$ : It is the same on any two space-like hypersurfaces that intersect the spacetime region in which $S_{i}$ is located. If the probabilities of the possible values of $R_{1}$ and $R_{2}$ are to satisfy the Relativistic Born Rule on the hypersurfaces $\alpha, \beta, \gamma$ and $\delta$, there must be a joint probability distribution, Prob, over the values of $R_{1}$ at $x_{1}$ and at $y_{1}$ and the values of $R_{2}$ at $x_{2}$ and at $y_{2}$, which yields as marginals the (single-time) Born-like probabilities for the values of $R_{1}$ and $R_{2}$ on all the four hypersurfaces. That is, let $R_{i}\left(x_{i}\right)$ $\left(R_{i}\left(y_{i}\right)\right)$ be the variable that corresponds to the value of the variable $R_{i}$ at $x_{i}\left(y_{i}\right), R_{1}\left(x_{1}\right)=a\left(R_{1}\left(y_{1}\right)=a\right)$ be the proposition that the value of $R_{1}$ at $x_{1}\left(y_{1}\right)$ is $a$, and $R_{2}\left(x_{2}\right)=b\left(R_{2}\left(y_{2}\right)=b\right)$ be the proposition that the value of $R_{2}$ at $x_{2}\left(y_{2}\right)$ is $b$, where $a$ takes either the value $r_{1}+$ or $r_{1}-$, and $b$ takes either the value $r_{2}+$ or $r_{2}-$. Let also $P_{R_{1}}\left(R_{1}\left(x_{1}\right)=a\right), P_{R_{2}}\left(R_{2}\left(x_{2}\right)=b\right)$, $P_{R_{1}}\left(R_{1}\left(y_{1}\right)=a\right)$ and $P_{R_{2}}\left(R_{2}\left(y_{2}\right)=b\right)$ be the projections onto the eigenspaces $R_{1}\left(x_{1}\right)=a, R_{2}\left(x_{2}\right)=b, R_{1}\left(y_{1}\right)=a$ and $R_{2}\left(y_{2}\right)=b$, respectively. Finally, let $i$ and $k$ each be a variable that takes either the value $r_{1}+$ or $r_{1}-$, and 
$j$ and $l$ each be a variable that takes either the value $r_{2}+$ or $r_{2}-$. Then, the probability distribution Prob must satisfy the following equalities for the joint probabilities of the values of $R_{1}$ and $R_{2}$ on $\alpha, \beta, \gamma$ and $\delta$ :

$$
\begin{aligned}
& \sum_{k, l} \operatorname{Prob} \quad\left(R_{1}\left(x_{1}\right)=a, R_{2}\left(x_{2}\right)=b, R_{1}\left(y_{1}\right)=k, R_{2}\left(y_{2}\right)=l\right)= \\
& =\operatorname{Tr}\left[P_{R_{1}}\left(R_{1}\left(x_{1}\right)=a\right) P_{R_{2}}\left(R_{2}\left(x_{2}\right)=b\right) \varphi(\alpha)\right] \\
& \sum_{i, j} \operatorname{Prob} \quad\left(R_{1}\left(x_{1}\right)=i, R_{2}\left(x_{2}\right)=j, R_{1}\left(y_{1}\right)=a, R_{2}\left(y_{2}\right)=b\right)= \\
& =\operatorname{Tr}\left[P_{R_{1}}\left(R_{1}\left(y_{1}\right)=a\right) P_{R_{2}}\left(R_{2}\left(y_{2}\right)=b\right) \varphi(\beta)\right] \\
& \sum_{i, l} \operatorname{Prob}\left(R_{1}\left(x_{1}\right)=i, R_{2}\left(x_{2}\right)=b, R_{1}\left(y_{1}\right)=a, R_{2}\left(y_{2}\right)=l\right)= \\
& =\operatorname{Tr}\left[P_{R_{1}}\left(R_{1}\left(y_{1}\right)=a\right) P_{R_{2}}\left(R_{2}\left(x_{2}\right)=b\right) \varphi(\gamma)\right] \\
& \sum_{j, k} \operatorname{Prob}\left(R_{1}\left(x_{1}\right)=a, R_{2}\left(x_{2}\right)=j, R_{1}\left(y_{1}\right)=k, R_{2}\left(y_{2}\right)=b\right)= \\
& =\operatorname{Tr}\left[P_{R_{1}}\left(R_{1}\left(x_{1}\right)=a\right) P_{R_{2}}\left(R_{2}\left(y_{2}\right)=b\right) \varphi(\delta)\right]
\end{aligned}
$$

where, as before, $|\varphi(\alpha)\rangle,|\varphi(\beta)\rangle,|\varphi(\gamma)\rangle$ and $|\varphi(\delta)\rangle$ are the states of $S_{1}+$ $S_{2}+A_{1}+A_{2}$ on $\alpha, \beta, \gamma$ and $\delta$, respectively. If Prob existed, it would satisfy certain Bell-type inequalities concerning the joint probabilities in (8). But since some of these inequalities are violated by orthodox quantum mechanics in the states (3)-(6), the Relativistic Born Rule fails. 
Here is an example of such violation. Suppose that on $\alpha R_{1}$ and $R_{2}$ have the values $\left(r_{1}+, r_{2}+\right)$. Since, by assumption, $R_{1}$ is a local property of $S_{1}$, it must have the same value on the hypersurface $\delta$. Thus, it follows from the Relativistic Born Rule that the probability that $R_{1}$ and $R_{2}$ have the values $\left(r_{1}+, r_{2}-\right)$ on $\delta$ is one. Since $R_{2}$ is a local property of $S_{2}$, if $S_{2}$ has the value $r_{2}-$ on $\delta$, it will have the value $r_{2}-$ on $\beta$ as well. And so the probability that $R_{2}$ has the value $r_{2}-$ on $\beta$ given that $R_{1}$ and $R_{2}$ have the values $\left(r_{1}+, r_{2}+\right)$ on $\alpha$ is one. A parallel argument leads to the conclusion that if $R_{1}$ and $R_{2}$ have the values $\left(r_{1}+, r_{2}+\right)$ on $\alpha$, the probability that $R_{1}$ will have the value $r_{1}-$ on $\beta$ is also one. Accordingly, if $R_{1}$ and $R_{2}$ have the values $\left(r_{1}+, r_{2}+\right)$ on $\alpha$, the probability that these quantities have the values $\left(r_{1}-, r_{2}-\right)$ on $\beta$ is one. But, by the Relativistic Born Rule, $|\varphi(\beta)\rangle$ assigns zero probability to these values. Therefore, it is impossible to satisfy the Relativistic Born Rule for the values of $R_{1}$ and $R_{2}$ on the hypersurfaces $\alpha, \beta, \gamma$ and $\delta$.

More generally, Myrvold's argument is that in the property assignments of current modal interpretations, the probabilities of local, possessed properties cannot be given by Born-like probabilities along every foliation of spacetime 
for any arbitrary initial quantum state. ${ }^{4}$

\section{WAYS OUT}

In response to Myrvold's no-go theorem, one may object to the presupposition that the Relativistic Born Rule is a necessary condition for relativistic modal interpretations. This presupposition seems to rely on the natural assumption that the probabilities of possessed properties should invariably be equal to the Born probabilities of these properties. But one may reject this assumption and insist that only the probabilities of observed properties should be equal to the Born probabilities on all spacelike hypersurfaces. The challenge is then to develop a relativistic dynamics that will violate the Relativistic Born Rule yet reproduce (at least approximately) the Born probabilities for observed properties, so that the empirical predictions for such properties will (in effect) be indistinguishable from the corresponding predictions of orthodox quantum mechanics.

\footnotetext{
${ }^{4}$ The scope of Myrvold's theorem is not limited only to current modal interpretations. It may also be applicable to other no-collapse theories that admit local properties of the type discussed above.
} 
In what follows, we shall focus on a different strategy for circumventing Myrvold's theorem, which involves a violation of some of the theorem's other premises. Recall that the theorem applies to local properties. It demonstrates that if the property assignments of current modal interpretations were to be compatible with relativity, the joint distribution of such properties would be constrained by Bell-type inequalities, and accordingly would deviate from the quantum-mechanical statistics. More particularly, the theorem demonstrates that the joint probabilities of the values of $R_{1}$ and $R_{2}$ on the hypersurfaces $\alpha$, $\beta, \gamma$ and $\delta$ will satisfy certain Bell-type inequalities if the following conditions obtain:

(i) Locality. The value of an observable $R_{i}$ is the same on any two hypersurfaces that intersect $x_{i}\left(y_{i}\right)$.

(ii) Joint Probability. The values of $R_{1}$ and $R_{2}$ have definite joint probabilities on the hypersurfaces $\alpha, \beta, \gamma$ and $\delta$.

But, granted the Relativistic Born Rule, these inequalities are violated in the states $|\varphi(\alpha)\rangle,|\varphi(\beta)\rangle,|\varphi(\gamma)\rangle$ and $|\varphi(\delta)\rangle$ in (3)-(6). 
This suggests that Myrvold's theorem will not be applicable to modal interpretations that satisfy the Relativistic Born Rule but violate Locality or Joint Probability in the set up of Myrvold's theorem. In the next section, we shall sketch an outline of a modal interpretation that violates both Locality and Joint Probability. In contrast to current modal interpretations, in this interpretation quantum-mechanical states assign only relational properties: Properties of systems are assigned only relative to other systems. Formally, one may modify the current non-relational modal interpretations, so as to yield a dynamics that violates Locality. But, in the context of these theories such modification would be rather ad hoc. By contrast, as we shall see below, the relational modal interpretation provides a natural ontological framework for the violation of these conditions.

The relational modal interpretation also solves other problems of current modal interpretations. First, a number of these interpretations violate the following conditions about the relation between the properties of composite systems and the properties of their subsystems:

Let $\mathcal{H}^{1}$ and $\mathcal{H}^{2}$ be the Hilbert spaces of the systems $S_{1}$ and $S_{2}$, 
respectively. Let $Q_{1}$ be an observable that pertains to $\mathcal{H}^{1}$ and $q$ be one of its values, let $P_{Q_{1}}(q)$ be a projection onto the eigenspace $Q_{1}=q$, and let $I_{2}$ be the identity operator for $\mathcal{H}^{2}$. Then:

Property Composition. If $S_{1}$ has the property (associated with) $P_{Q_{1}}(q)$, then $S_{1}+S_{2}$ has the property (associated with) $P_{Q_{1}}(q) \otimes$ $I_{2}$.

Property Decomposition. If $S_{1}+S_{2}$ has the property (associated with) $P_{Q_{1}}(q) \otimes I_{2}$, then $S_{1}$ has the property (associated with) $P_{Q_{1}}(q)$

The violation of these conditions is inexplicable in non-relational modal interpretations. $^{5}$ Yet, as we shall see in Sec. 5.1, it is naturally explained in the relational modal interpretation.

\footnotetext{
${ }^{5}$ Two comments: (i) The perspectivalist modal interpretations of $\operatorname{Kochen}^{(7)}$ and Bene and Dieks ${ }^{(8)}$ are exceptions. (ii) The violation of Property Composition and Property Decomposition is the main motivation for developing the atomic modal interpretations, where these conditions are satisfied by construction.
} 
Second, the Schmidt-decomposition and the spectral-resolution modal interpretations (see Sec. 2) fail to recover our experience in certain decoherence situations, and accordingly fall short of providing a satisfactory solution to the measurement problem. In Berkovitz and Hemmo ${ }^{(23)}$, we argue that this problem does not arise in the relational modal interpretation.

Third, all current modal interpretations face the challenge of explaining why properties are assigned only in preferred bases (e.g. the Schmidt bases in the Schmidt-decomposition and the Spectral-resolution modal interpretations). As we shall see in the next section, the relational modal interpretation postulates no preferred basis: Properties are assigned in all bases.

\section{AN OUTLINE OF A RELATIONAL MODAL INTERPRETA- TION}

We now turn to present the relational modal interpretation. We start with the property and probability assignments (Sec. 5.1), then turn to outline the dynamics of probabilities (Sec. 5.2), and conclude by explaining how the 
relational modal interpretation circumvents Myrvold's no-go theorem (Sec. 5.3).

\subsection{The property assignment}

The general idea of the property assignment in the relational modal interpretation is that one carves up the universe into two systems, $S_{I}$ and $S_{I I}$, and assigns properties to $S_{I}$ relative to $S_{I I}$ according to $S_{I}$ 's reduced state (obtained by partial tracing of the state of $S_{I}+S_{I I}$ over the Hilbert space of $\left.S_{I I}\right)$. Each partition of the universe defines a context $S_{I I}$ relative to which a subset of $S_{I}$ 's relational properties is defined. There is no fundamental partition of the universe that is somehow preferred. All partitions are ontologically on equal footing: Each partition picks out a subset of relational properties.

In more detail: For any (normalized) quantum state of the universe, for any partition of the universe into systems $S_{I}$ and $S_{I I}$, and for any orthonormal basis for the Hilbert space associated with $S_{I}$, the range of the possible 
properties of $S_{I}$ relative to $S_{I I}$ is given by $S_{I}$ 's reduced state. In any such orthonormal basis, the range of $S_{I}$ 's possible properties relative to $S_{I I}$ is given by the projections corresponding to the non-zero diagonal elements of $S_{I}$ 's reduced state. ${ }^{6}$ The properties that $S_{I}$ has relative to $S_{I I}$ are decomposable into the properties that subsystems of $S_{I}$ have relative to $S_{I I}$. That is, let $S_{I}^{*}$ and $S_{I}^{* *}$ be any partition of $S_{I}$, let $\mathcal{H}^{1}$ and $\mathcal{H}^{2}$ be the Hilbert spaces associated with $S_{I}^{*}$ and $S_{I}^{* *}$ respectively, let $P$ be an $\mathcal{H}^{1}$ observable and $Q$ be an $\mathcal{H}^{2}$ observable, and let $p$ and $q$ be values of $P$ and $Q$ respectively. If $S_{I}$ has the property $P=p$ and $Q=q$ relative to $S_{I I}$, then $S_{I}^{*}$ (as a subsystem of $S_{I}$ ) has the property $P=p$ relative to $S_{I I}$ and $S_{I}^{* *}$ has (as a subsystem of $\left.S_{I}\right)$ the property $Q=q$ relative to $S_{I I}$.

Reduced states of systems do not only prescribe the range of the possible relational properties of a system, they also prescribe the single-time probabilities of these properties. The on-diagonal elements of the reduced state

\footnotetext{
${ }^{6}$ Note that this means that in contrast to current modal interpretations, in the re-
} lational modal interpretation the properties of $S_{I}$ are assigned in any resolution of the reduced state of $S_{I}$, and not only in its spectral resolution! For more details about this issue, see Berkovitz and Hemmo ${ }^{(23)}$. 
of $S_{I}$ provide the single-time probabilities of the properties of $S_{I}$ (and its subsystems) relative to $S_{I I}$. Properties that are defined relative to the same context, i.e. the same systems, have definite joint probabilities. For example, if $S_{I}^{*}$ and $S_{I}^{* *}$ is a partition of $S_{I}$, the properties that $S_{I}^{*}$ and $S_{I}^{* *}$ may have relative to $S_{I I}$ have definite joint probabilities. On the other hand, properties that are defined relative to different contexts are unrelated to each other and accordingly have no definite joint probabilities. This is required in order to avoid the no-hidden-variables theorems, such as Kochen and Specker's ${ }^{(24)}$. But it is also a direct consequence of the above property assignment. In the relational modal interpretation, the single-time probabilities are given by reduced states, and there are no reduced states to provide joint probabilities for properties that are related to different contexts. Consider, for instance, the probability that $S_{I}^{*}$ has the property $P=p$ relative to $S_{I}^{* *}+S_{I I}$, the probability that $S_{I}^{* *}$ has the property $Q=q$ relative to $S_{I}^{*}+S_{I I}$ and the joint probability of these properties. The probability that $S_{I}^{*}$ has the property $P=p$ relative to $S_{I}^{* *}+S_{I I}$ is given by the reduced state of $S_{I}^{*}$, the probability that $S_{I} * *$ has the property $Q=q$ relative to $S_{I}^{*}+S_{I I}$ is given by the reduced state of $S_{I}^{* *}$. But, the joint probability of these properties is not given by the reduced state of $S_{I}\left(S_{I}^{*}+S_{I}^{* *}\right)$ or any other reduced state; 
for the reduced state of $S_{I}$ only gives the joint probability of the properties that $S_{I}^{*}$ and $S_{I}^{* *}$ each has relative to $S_{I I}$.

Four remarks about the nature and range of relational properties: First, note that in the relational modal interpretation the properties that $S_{I}$ has relative to $S_{I I}$ are related to $S_{I I}$ simpliciter rather than to $S_{I I}$ having particular properties. This type of relational properties is in a sense 'thinner' than the relational properties postulated by Everett-like relative-state interpretations $^{(9,12,13)}$, where the properties of $S_{I}$ are related to the properties of $S_{I I}$. Second, note that in the relational modal interpretation the range of the possible properties of $S_{I}$ relative to $S_{I I}$ and the single-time probabilities of these properties depend only on $S_{I}$ 's reduced state. This means that if there is no change in this reduced state, there can be a change in neither the range of $S_{I}$ 's possible properties relative to $S_{I I}$, nor their single-time probabilities. Yet, as we shall see in Sec. 5.2, in some of the most distinctively characteristic quantum situations the actual properties that a subsystem of $S_{I}$ has relative to $S_{I I}$ may change even when the reduced state of this subsystem remains the same. Third, as is easily seen from the above property assignment, both the properties that a system actually has and the proper- 
ties that it may have will generally be different relative to different systems (contexts). Fourth, although Property Composition and Property Decomposition (see Sec. 4) fail, their failure does not raise any explanatory difficulty. In the relational modal interpretation, this failure reflects the fact that in certain quantum-mechanical states the range of the possible properties of a system will be different relative to different systems (contexts).

\subsection{THE DYNAMICS}

The universal dynamics of relational properties may be described as a sum average over the dynamics in two extreme cases: (i) the dynamics in cases of no entanglement, and (ii) the dynamics in cases of maximal entanglement.

Before we turn to outline the dynamics in these extreme cases, we should first introduce the relevant notion of entanglement in play. The entanglement we have in mind is bipartite, i.e. entanglement between two systems. Its mea-

sure may be defined in various ways. One measure, proposed by Shimony ${ }^{(25)}$, is defined in terms of the minimal distance in Hilbert space norm between the 
quantum state of the entangled systems and the set of all possible product states of these systems. That is, if the state of the composite system $S 1+S 2$ is $|\psi\rangle$, the degree of entanglement between $S_{1}$ and $S_{2}$ is the minimized (normalized) distance between $|\psi\rangle$ and the set of all the product states of $S_{1}$ and $S_{2}$. A related measure of degree of entanglement, proposed by Abouraddy et al. ${ }^{(26)}$, is defined in terms of the distance in Hilbert space norm between $|\psi\rangle$ and both a maximally entangled state $\left|\psi_{e}\right\rangle$ and a factorizable (product) state $\left|\psi_{f}\right\rangle$ orthogonal to it, such that $|\psi\rangle$ is a normalized superposition of $\left|\psi_{e}\right\rangle$ and $\left|\psi_{f}\right\rangle$. But, other measures of degree of entanglement may also be applicable.

The above notions of entanglement apply to pure states. But, in general we shall need to quantify the degree of entanglement between systems in mixed states (obtained from pure states by partial tracing). This raises no particular problems, as all the characteristic bipartite measures of degree of entanglement for pure states are also applicable to mixed states. In particular, the above geometrical measures of entanglement are easily generalized to mixed states. Consider, for example, the measure of entanglement as the minimal (normalized) distance from the set of all product states. In pure states, this distance is measured in Hilbert space norm, whereas in mixed 
states it is measured in the space of the self-adjoint operators. That is, let $B$ be any mixed state of the composite system $S_{1}+S_{2}$, and let $\mathbf{C}$ be the convex set of all the mixed product states of $S_{1}$ and $S_{2}$. The minimal distance between $B$ and $\mathbf{C}$ is proportional to the distance between $B$ and any state $A$ in $\mathbf{C}$ such that $\operatorname{Tr}(B \otimes A) \leq \operatorname{Tr}\left(B \otimes A^{\prime}\right)$ for any other state $A^{\prime}$ in $\mathbf{C}$.

In the context of the relational modal interpretation, the relevant systems for measuring the degree of entanglement are determined by the relational properties under consideration and the relevant transformations. Let $S_{I}$ and $S_{I I}$ be a partition of the universe, and $S_{I}^{*}$ and $S_{I}^{* *}$ be a partition of $S_{I}$, and $S_{I I}^{*}$ be a subsystem of $S_{I I}{ }^{7}$ Let $U$ be any unitary transformation on the state of $S_{I}^{*}+S_{I I}^{*}$ and the identity transformation on the state of all the other subsystems of $S_{I}+S_{I I}$. The degree of entanglement relevant for the dynamics of the properties of $S_{I}$ and its subsystems relative to $S_{I I}$ under the transformation $U$ is determined by the degree of entanglement between $S_{I}^{* *}$ and $S_{I}^{*}+S_{I I}^{*}$ in the initial state of $S_{I}^{* *}+S_{I}^{*}+S_{I I}^{*}$ (namely, the state that this composite system has before $U$ is applied). And the effect that $U$ has on these

\footnotetext{
${ }^{7}$ Here, by 'a subsystem of $S_{I I}$ ' we mean any subsystem of it, including $S_{I I}$ itself and
} the null system. 
properties depends on this degree of entanglement. The general idea is that when systems are entangled, local transformations have global effect. The dynamics of the relational properties of $S_{I}^{* *}$ depends (among other things) on the entanglement between $S_{I}^{*}+S_{I I}^{*}$ and $S_{I}^{*}$.

Consider, for example, the Hadamard transformation on the state of $S_{2}+$ $A_{2}$ (i.e. the Hadamard transformation on the eigenstates of $R_{2} \otimes P_{2}$ ) and the identity transformation on the state of $S_{1}+A_{1}$ ) between the hypersurfaces $\alpha$ and $\delta$, and the properties that $S_{1}$ and $S_{2}$ have relative to $A_{1}+A_{2}$ in Myrvold's set up (see Sec. 3). Applying our general rule to this specific case, $S_{I}^{* *}$ is $S_{1}, S_{I}^{*}$ is $S_{2}, S_{I I}^{*}$ is $A_{2}$, and the relevant degree of entanglement is the one between $S_{1}$ and $S_{2}+A_{2}$ in the (reduced) state of $S_{1}+S_{2}+A_{2}$ on $\alpha$ (obtained by partial tracing from $|\varphi(\alpha)\rangle)$. The degree of entanglement between $S_{1}$ and $S_{2}+A_{2}$ may for example be measured by the minimal (normalized) distance in the space of the (self adjoint) operators between this (reduced) state and the convex set of all the (reduced) product states of $S_{1}$ and $S_{2}+A_{2}$.

Given the above measure of degree of entanglement, let us now consider the dynamics in the extreme cases of maximal entanglement and no entan- 
glement. Consider, first, the case of no entanglement. Let $U$ be a unitary transformation on the state of $S_{I}+S_{I I}$. If the reduced state of $S_{I}$ does not change under $U$, the relational properties of $S_{I}$ relative to $S_{I I}$ do not change. If the reduced state of $S_{I}$ changes, then the probabilities of the properties that $S_{I}$ may have relative to $S_{I I}$ depend on the properties that $S_{I}$ has relative to $S_{I I}$ and the transformation $U$. The properties of $S_{I}$ associated with projectors that commute with $U$ evolve deterministically, so as to return the single-time Born-like probabilities; and the properties of $S_{I}$ associated with projectors that do not commute with $U$ evolve indeterministically, so as to return the single-time Born-like probabilities.

While the above transition probabilities resemble the probabilities obtained by a sequential application of the Born rule in a collapse theory, the dynamics in cases of maximal entanglement is very different. The transition probabilities of $S_{I}$ 's properties relative to $S_{I I}$ induced by a transformation $U$ on the state of $S_{I}+S_{I I}$ are directly proportional to the distance between the (reduced) states of $S_{I}$ before and after applying $U$ and the single-time Born-like probabilities of $S_{I}$ 's properties relative to $S_{I I}$ after applying $U$. That is, let the reduced states of $S_{I}$ before and after applying $U$ be $\rho_{i}$ and 
$\rho_{f}$, respectively. Let $Q$ and $R$ be two observables that pertain to $S_{I}$, and let $q$ and $r$ be values of $Q$ and $R$, respectively. Then, the probability that $S_{I}$ has the property $Q=q$ relative to $S_{I I}$ in the state $\rho_{f}$, given that it has the property $R=r$ relative to $S_{I I}$ in the state $\rho_{i}$ is equal to (the (normalized) distance between $\rho_{i}$ and $\rho_{f}$ ) times (the single-time Born-rule probability of $Q=q$ in the state $\left.\rho_{f}\right)$

It is noteworthy that both the dynamics in cases of maximal entanglement and the dynamics in cases of no entanglement are holistic in nature. The transition probabilities of the properties that $S_{I}^{*}$ has, as a subsystem of $S_{I}$, relative to $S_{I I}$ (generally) depend on the properties that $S_{I}$ has relative to $S_{I I}$ and not only on the properties that $S_{I}^{*}$ has relative to $S_{I I}$.

The universal dynamics is a weighted average of the dynamics in cases of no entanglement and the dynamics in cases of maximal entanglement. That is, let $|\psi\rangle$ be the state of the universe, $S_{I}$ and $S_{I I}$ be any partition of the universe, $S_{I}^{*}$ and $S_{I}^{* *}$ be any partition of $S_{I}$, and $S_{I I}^{*}$ be a subsystem of $S_{I I}$. Let $U$ be any unitary transformation on the state of $S_{I}^{*}+S_{I I}^{*}$ and the identity transformation on the state of all the other subsystems of $S_{I}+S_{I I}$. Let $d(e)$ 
be the degree of entanglement between $S_{I}^{*}+S_{I I}^{*}$ and $S_{I}^{* *}$ in the state $|\psi\rangle$. Let $P(Q=q / R=r)$ be the probability that $S_{I}$ has the property $Q=q$ relative to $S_{I I}$ in the state $U|\psi\rangle$ given that it has the property $R=r$ relative to $S_{I I}$ in the state $|\psi\rangle$, and let $P_{M E}(Q=q / R=r), P_{N E}(Q=q / R=r)$ and $P_{U}(Q=q / R=r)$ denote respectively the value of $P(Q=q / R=r)$ according to the dynamics in cases of maximal entanglement, the dynamics in cases of no entanglement and the universal dynamics. Let $d(s)$ be the (normalized) distance between the reduced states of $S_{I}$ in the states $|\psi\rangle$ and $U|\psi\rangle$. Then:

$$
\begin{aligned}
P_{U}(Q=q / R=r)= & d(e) \cdot d(s) \cdot P_{M E}(Q=q / R=r)+ \\
& +(1-d(e) \cdot d(s)) \cdot P_{N E}(Q=q / R=r) .
\end{aligned}
$$

If the distribution of properties is given by the single-time Born-like probabilities on any spacelike hypersurface, then (by construction) the dynamics (i.e. the transition probabilities) in cases of no entanglement and in cases of maximal entanglement will each reproduce the single-time Born-like probabilities on any other spacelike hypersurface. Accordingly, the universal dynamics (9) will also reproduce the single-time Born-like probabilities. 


\subsection{Myrvold's theorem revisited}

Having completed the outline of the relational modal interpretation, we now turn to show how this interpretation circumvents Myrvold's theorem. Con-

sider again Myrvold's set up. In the states $|\varphi(\alpha)\rangle,|\varphi(\beta)\rangle,|\varphi(\gamma)\rangle$ and $|\varphi(\delta)\rangle$ in (3)-(6) the value of $R_{1}$, as a property of $S_{1}$, relative to $S_{2}+A_{1}+A_{2}$ is either $r_{1}+$ or $r_{1}-;$ and the value of $R_{2}$, as a property of $S_{2}$, relative to $S_{1}+A_{1}+A_{2}$ is either $r_{2}+$ or $r_{2}-$. Since these values are related to different contexts, their joint probabilities do not exist. Accordingly, Myrvold's theorem does not apply to these properties.

Consider alternatively the range of the possible values that $R_{1}$ and $R_{2}$ have, as properties of the composite system $S_{1}+S_{2}$, relative to $A_{1}+A_{2}$. In the states (3)-(6), these relational values of $R_{1}$ and $R_{2}$ are definite and, moreover, they relate to the same context and accordingly have joint probabilities. But, since $S_{2}+A_{2}$ is entangled with $S_{1}$ and $S_{1}+A_{1}$ is entangled with $S_{2}$ (see Sec. 5.2 ), the value of $R_{1}$, as a property of $S_{1}+S_{2}$, relative to $A_{1}+A_{2}$ may not be the same on $\alpha$ and $\delta$ (i.e. in the states $|\varphi(\alpha)\rangle$ and $|\varphi(\delta)\rangle$ ) and on $\gamma$ and $\beta$ (i.e. in the states $|\varphi(\gamma)\rangle$ and $|\varphi(\beta)\rangle$ ); and the value of $R_{2}$, as a property 
of $S_{1}+S_{2}$, relative to $A_{1}+A_{2}$ may not be the same on $\alpha$ and $\gamma$ (i.e. in the states $|\varphi(\alpha)\rangle$ and $|\varphi(\gamma)\rangle$ ) and on $\delta$ and $\beta$ (i.e. in the states $|\varphi(\delta)\rangle$ and $|\varphi(\beta)\rangle$ ). (Indeed, these degrees of entanglement are substantial. Accordingly, the chance that the value of $R_{1}$ as a property of $S_{1}+S_{2}$ relative to $A_{1}+A_{2}$ will not be the same on $\alpha$ and $\delta(\gamma$ and $\beta)$, and the chance that the value of $R_{2}$ as a property of $S_{1}+S_{2}$ relative to $A_{1}+A_{2}$ will not be the same on $\alpha$ and $\gamma(\delta$ and $\beta)$, are both significant.) Thus, Myrvold's theorem is inapplicable to these relational values of $R_{1}$ and $R_{2}{ }^{8}$

Generalizing the above reasoning, it is not difficult to show that Myrvold's theorem is also inapplicable to any other relational values of $R_{1}$ and $R_{2}$ and, more generally, any other relational properties. Thus, we conclude that Myrvold's theorem is inapplicable to the relational modal interpretation.

${ }^{8}$ The fact that the value of $R_{1}$ relative to $A_{1}+A_{2}$ is not the same on $\alpha$ and $\delta$ ( $\gamma$ and $\beta$ ) is sufficient for circumventing Myrvold's theorem. But in order to avoid other possible no-go theorems for relativistic modal interpretations, it is important that the chance that this value of $R_{1}$ will not be the same on $\alpha$ and $\delta(\gamma$ and $\beta$ ) will be proportional to the degree of entanglement between $S_{2}+A_{2}$ and $S_{1}$ on $\alpha(\gamma)$; and similarly, mutatis mutandis, for the value of $R_{2}$ relative to $A_{1}+A_{2}$. 
It may be objected that the relational modal interpretation postulates that local properties may be hypersurface-dependent properties, which are frame-dependent properties in disguise. Here the idea may be that since the value of e.g. $R_{1}$ (as a property of $S_{1}+S_{2}$ ) relative to $A_{1}+A_{2}$ may not be the same on the hypersurfaces $\alpha$ and $\delta$, this property is hypersurface dependent. Thus, as each of these hypersurfaces could be associated with a different frame of reference, it may be argued that this relational value of $R_{1}$ is really a frame-dependent property in disguise.

In reply, it is important to stress that the values of $R_{1}$ and $R_{2}$ (as properties of $\left.S_{1}+S_{2}\right)$ relative to $A_{1}+A_{2}$ are not hypersurface dependent per se, at least not if by hypersurface dependent properties it is meant properties that are defined relative to hypersurfaces. The dynamics of the value of $R_{1}$ (as a property of $\left.S_{1}+S_{2}\right)$ relative to $A_{1}+A_{2}$ depends on the degree of entanglement between $S_{2}+A_{2}$ and $S_{1}$ and the transformation of the states of $S_{2}+A_{2}$; and similarly, mutatis mutandis, for the value of $R_{2}$ (as a property of $S_{1}+S_{2}$ ) relative to $A_{1}+A_{2}$. Thus, these values are highly nonlocal properties. But, they are not hypersurface dependent per se: They are not defined relative to hypersurfaces. Indeed, the relational value of e.g. $R_{1}$ relative to $A_{1}+A_{2}$ will 
be the same on the hypersurfaces $\alpha$ and $\delta$ if the Hadamard transformation on the eigenstates of $R_{2} \otimes P_{2}$ is substituted by an identity transformation. It is also noteworthy that there is a conceptual difference between framedependent and hypersurface-dependent properties ${ }^{(27-32)}$ : Properties that are hypersurface dependent may be frame independent.

In any case, as is not difficult to see the relational values of $R_{1}$ and $R_{2}$ (as properties of $S_{1}+S_{2}$ ) relative to $A_{1}+A_{2}$ are invariant across all inertial reference frames, and accordingly are frame independent. Moreover, unlike the Bacciagaluppi and Dickson ${ }^{(21)}$ dynamics for the Vermaas-Dieks modal interpretation, and the dynamics in Bohm's theory ${ }^{(20)}$ and in the GRW/Pearle collapse models ${ }^{(33,34)}$, the dynamics of properties in the relational modal interpretation does not pick out any preferred reference frame. Thus, the objection above is ungrounded.

\section{ON EXPERIENCE IN THE RELATIONAL} MODAL INTERPRETATION 
In accounting for experience, the relational modal interpretation face two main challenges. First, there is the challenge from e-nonlocality. Recall (Sec. 5.2-5.3) that the relational modal interpretation postulates that in cases of entanglement the value of apparently local quantities may be different on two different spacelike hypersurfaces that intersect the spacetime region in which the system they pertain to is located. Yet, our experience seems to suggest that this type of nonlocality never occurs.

Secondly, there is the challenge from multiplicity. Similarly to any other physical object, the brain of a human observer has many different relational properties, i.e. properties that are related to different contexts (systems). Given that properties that are related to different contexts are uncorrelated, it seems plausible to assume that our beliefs about the physical objects that appear in our experience are associated with brain properties that are related to a single context. Yet, there are many possible contexts. So the question arises as to which of them accounts for our beliefs.

We shall address first the challenge from multiplicity, then the challenge from e-nonlocality. We believe that given current knowledge of the brain 
and mind-brain relationships, the best one could do is to demonstrate that it is possible to establish systematic correlations between brain properties, physical properties that appear in our experience and our beliefs about them. The basic reasoning is as follows. It is possible to show that brain properties that are related to certain contexts could systematically be correlated with properties of physical objects that are associated with our experience. Thus, granted the common assumption of systematic correlations between brain states and states of mind, it is possible to establish systematic correlations between brain properties, properties of physical objects and beliefs about them.

To spell out this reasoning in some more detail, consider a toy universe that contains only two observers, $O_{1}$ and $O_{2}$. Let $S_{I}$ comprise a particle $P$, a measuring apparatus $M$, and the observers $O_{1}$ and $O_{2}$, and let $S_{I I}$ be the rest of the universe. Suppose, for the sake of simplicity, that $M$ performs an ideal $z$-spin measurement on $P$, and $O_{1}$ and $O_{2}$ both observe the measurement outcome, and accordingly the state of $S_{I}\left(P+M+O_{1}+O_{2}\right)$ and $S_{I I}$ is the following:

$$
|\psi\rangle=\sum_{i, j, k, l, m} c_{i, j, k, l, m}\left|\alpha_{i}\right\rangle_{P}\left|\beta_{j}\right\rangle_{M}\left|\gamma_{k}\right\rangle_{O_{1}}\left|\delta_{l}\right\rangle_{O_{2}}\left|\lambda_{m}\right\rangle_{S_{I I}}
$$


where $\alpha_{i}$ ranges over $z+\left(z\right.$-spin 'up') and $z-\left(z\right.$-spin 'down'), $\beta_{j}$ ranges over 'up' (pointer pointing to 'up') and 'down' (pointer pointing to 'down'), $\gamma_{k}$ and $\delta_{l}$ each ranges over 'b-up' ('believe up') and 'b-down' ('believe down') and $\lambda_{m}$ ranges over $\lambda_{1}$ and $\lambda_{2}$. There always exists a normalized basis $\left\{\left|r_{1}\right\rangle,\left|r_{2}\right\rangle\right\}$ in the Hilbert space associated with $S_{I I}$, such that $|\psi\rangle$ can be rewritten as follows:

$$
\begin{aligned}
|\psi\rangle= & c_{1}|z+\rangle_{P}|\mathrm{up}\rangle_{\mathrm{M}}|\mathrm{b}-\mathrm{up}\rangle_{\mathrm{O}_{1}}|\mathrm{~b}-\mathrm{up}\rangle_{\mathrm{O}_{2}}\left|\mathrm{r}_{1}\right\rangle_{\mathrm{S}_{\mathrm{II}}}+ \\
& \left.\left.\left.+c_{2}|z-\rangle_{P} \mid \text { down }\right\rangle_{\mathrm{M}} \mid \mathrm{b}-\text { down }\right\rangle_{\mathrm{O}_{1}} \mid \mathrm{b}-\text { down }\right\rangle_{\mathrm{O}_{2}}\left|\mathrm{r}_{2}\right\rangle_{\mathrm{S}_{\mathrm{II}}}
\end{aligned}
$$

where $\left|r_{1}\right\rangle_{S_{I I}}$ and $\left|r_{2}\right\rangle_{S_{I I}}$ are not necessarily orthogonal. Since the properties of $S_{I}$ (i.e. of $P+M+O_{1}+O_{2}$ ) relative to $S_{I I}$ are given by $S_{I}$ 's reduced state, the particle, the pointer and the brains of the two observers have definite relational properties that are appropriately correlated with each other. Suppose, for example, that relative to $S_{I I}$ the particle has (as a subsystem of $\left.S_{I}\right) z$-spin 'up'. Then, relative to $S_{I I}$ the position of the apparatuss pointer (as a subsystem of $S_{I}$ ) is 'up' and the brain properties of both observers (as subsystems of $S_{I}$ ) are those of 'b-up'.

One could easily generalize this example to show that brain properties of different observers that are related to the same context would be appro- 
priately correlated with each other and with the physical properties that appear in our experience. Thus, if any of these subsets of relational brain properties are correlated with the corresponding states of mind, there will be systematic correlations between a certain subset of relational properties of physical systems and observers' beliefs about these systems. ${ }^{9}$ But, since subsets of properties that are related to different contexts are uncorrelated, the question is which subset of relational brain properties is correlated with our beliefs about the physical properties that appear in our experience. We think that the question of which brain properties are systematically correlated to such beliefs is not unique to the relational modal interpretation, yet due to proliferation of definite properties this question appears to be more acute in the context of this interpretation.

Turning to the challenge from e-nonlocality, we shall argue below that the dynamics of properties in the relational modal interpretation renders the e-nonlocality postulated by this interpretation unobservable. Recall that

\footnotetext{
${ }^{9}$ It is not difficult to show that observers' experience could be accounted for even if they are related to different contexts. But, for want of space, we shall not discuss this issue here.
} 
e-nonlocality occurs when the value of an apparently local quantity is not the same on two hypersurfaces that intersect the region in which the system it pertains to is located. In order for e-nonlocality to be detectable, the properties of some macroscopic systems have to record it. This means that the recording macroscopic systems have to be entangled with the system the properties of which are e-nonlocal. But, it follows from the dynamical laws of the relational modal interpretation that the chance that e-nonlocality occurs in circumstances in which it could be so recorded is virtually zero for the following reason.

Macroscopic systems undergo decoherence interactions with their environment. In such interactions, the reduced states of these systems (obtained by tracing over the environment's degrees of freedom) will be very nearly diagonal in the so-called 'pointer bases,' i.e. the bases that are selected by the decoherence interaction. ${ }^{10}$ This means that in these bases the on-diagonal elements will correspond to approximately product states. In particular,

\footnotetext{
${ }^{10}$ More exactly, the observables corresponding to the 'pointer' bases approximately commute with the Hamiltonian of the interaction between these macroscopic systems and the environment.
} 
the reduced state of the composite macroscopic recording system and the recorded system will be very nearly diagonal in the 'recording' bases and thus correspond to approximately product states in these bases. In such product states the relevant degree of entanglement (as proposed in Sec. 5.2) turns out to be approximately zero. Accordingly, the properties of the recording and recorded systems relative to the environment will effectively evolve according to the dynamics in cases of no entanglement, where e-nonlocality does not occur.

But the dynamical laws of the relational modal interpretation are such that even if macroscopic recording systems were isolated from the environment, and accordingly e-nonlocality could occur in these systems and in the systems they record, such e-nonlocality would still be unobservable because of memory failure. To see why, consider again the set up of Myrvold's theorem, where the systems $S_{1}, S_{2}, A_{1}$ and $A_{2}$ are in the states (3)-(6) on the hypersurfaces $\alpha, \beta, \gamma$ and $\delta$ (see Sec. 3). Recall that in this set up, the pointer observable $P_{1}$ of the apparatus $A_{1}$ measures the value of the observable $R_{1}$ of the system $S_{1}$. Let $O_{1}$ be an observer that monitors the value of the pointer observable $P_{1}$, let $B_{1}$ be a brain observable associated with $O_{1}$ 's 
beliefs about the value of $P_{1}$, and let $M_{1}$ be a brain observable associated with $O_{1}$ 's memory of the value of $B_{1}$ on $\alpha \cdot{ }^{11}$ (To simplify terminology, $O_{1}$ will refer both to a physical system and to an agent. Context will distinguish between these different uses.) According to the dynamics of the relational modal interpretation outlined in Sec. 5.2, if $S_{1}, A_{1}, S_{2}, A_{2}$ and $O_{1}$ were isolated from the environment, some relational values of e.g. $P_{1}$ may not be the same on $\alpha$ and $\delta$, i.e. e-nonlocality may occur. Consider, for example, the value of $P_{1}$, as a property of $S_{1}+A_{1}+A_{2}+O_{1}$, relative to the rest of the universe. In the Hadamard transformation on the eigenstates of $R_{2} \otimes P_{2}$ between the hypersurfaces $\alpha$ and $\delta$, the transition probabilities are such that this value of $P_{1}$ may not be the same on $\alpha$ and $\delta$. In order to observe the difference between the values of $P_{1}$ on $\alpha$ and $\delta, O_{1}$ would have to reliably monitor and compare these values. To monitor and remember the value of $P_{1}$ on $\alpha$, the eigenstates of $B_{1}$ and $M_{1}$ will have to get correlated with the eigenstates of $P_{1}$. But, it follows from the above dynamics that in that case the values of $P_{1}, B_{1}$ and $M_{1}$, as properties of $S_{1}+A_{1}+A_{2}+O_{1}$, relative to the rest of the universe would be invariantly correlated. In particular, if

\footnotetext{
${ }^{11}$ Here, we make the substantial assumption of a strict association between brain observables and states of mind. But, we believe that the main thrust of our reasoning below will be valid even if such strict association does not exist.
} 
the values of $P_{1}$ and $B_{1}$ on $\alpha$ were different from their values on $\delta$, the value of $M_{1}$ would be similarly different on these hypersurfaces. Accordingly, the observer $O_{1}$ would not be able to notice that the value of $P_{1}$ on $\alpha$ is different from its value on $\delta$, and so would not be able to observe the e-nonlocality in this value and in the corresponding relational value of $R_{1}$ (i.e. the value of $R_{1}$, as a property of $S_{1}+A_{1}+A_{2}+O_{1}$, relative to the rest of the universe).

Indeed, some relational values of $M_{1}$, e.g. the value of $M_{1}$ as a property of $O_{1}$ relative to the rest of the universe, would be the same on $\alpha$ and $\delta$ even if $S_{1}, A_{1}, S_{2}, A_{2}$ and $O_{1}$ were isolated from the environment. But, this relational value of $M_{1}$ would be uncorrelated with the relational value of $P_{1}$ as a property of $S_{1}+A_{1}+A_{2}+O_{1}$ relative to the rest of the universe; for note that here 'the rest of the universe' refers to a different set of systems, namely all the systems except for $S_{1}, A_{1}, A_{2}$ and $O_{1}$ (rather than all the systems except for $O_{1}$ ). Thus, the value of $M_{1}$ as a property of $O_{1}$, relative to the rest of the universe on $\alpha$ cannot be considered as a memory of the value of $P_{1}$, as a property of $S_{1}+A_{1}+A_{2}+O_{1}$, relative to the rest of the universe on $\delta$. Accordingly, this value of $M_{1}$ cannot be used as a basis for detecting the e-nonlocality in the values of $P_{1}$ and $R_{1}$, as properties of $S_{1}+A_{1}+A_{2}+O_{1}$, 
relative to the rest of the universe.

More generally, any relational value of $P_{1}$ that is not the same on $\alpha$ and $\delta$ will be correlated with a relational value of $M_{1}$ that is similarly not the same on $\alpha$ and $\delta$, and be uncorrelated with all the other relational values of $M_{1}$. But, if a relational value of $M_{1}$ is correlated with a relational value of $P_{1}$, then this value of $M_{1}$ on $\delta$ will be a totally unreliable memory of the value of $P_{1}$ on $\alpha$; and if a relational value of $M_{1}$ is uncorrelated with a relational value of $P_{1}$, then this value of $M_{1}$ on $\delta$ will not be a memory of the value of $P_{1}$ on $\alpha$. Thus, in either case the value of $M_{1}$ is of no use for detecting e-nonlocality in the values of $P_{1}$ and $R_{1}$. Generalizing the above reasoning, it is possible to show that observers will have no way to detect any e-nonlocality in the value of quantities of microscopic or macroscopic systems. On the basis of similar considerations, it is also not difficult to show that the experiences of different observers associated with different reference frames (say, the reference frame in which $\alpha$ is a hyperplane of simultaneity and the reference frame in which $\delta$ is a hyperplane of simultaneity) will be invariably compatible with each other with respect to the results of any observation whatsoever. Thus, we conclude that in the relational modal interpretation 
the postulated e-nonlocality would in principle be unobservable even if the systems in question were completely isolated from their environment (and thus unaffected by decoherence interactions).

\section{CONCLUSIONS}

Modal interpretations of quantum mechanics were designed to solve the measurement problem and to reconcile quantum mechanics with the special theory of relativity. Some modal interpretations offer a solution to the measurement problem. But, as the no-go theorems by Dickson and Clifton ${ }^{(4)}$, Arntzenius $^{(5)}$ and Myrvold ${ }^{(6)}$ suggest, none of the current modal interpretations is compatible with relativity. In this paper, we considered Myrvold's theorem and proposed that a way to evade it is to reject its presupposition (embodied in the Relativistic Born Rule) that properties that are commonly thought of as local (such as a pointer's position) are indeed local and always have definite joint probabilities. ${ }^{12}$ We argued that the violation of this as-

\footnotetext{
12 Note that the rejection of this presupposition does not imply the rejection of the
} Relativistic Born Rule. Indeed, the relational modal interpretation trivially satisfies this condition, as all the properties postulated by this interpretation are nonlocal. 
sumption could naturally be obtained in a relational modal interpretation, which assigns only relational properties to systems. In this interpretation, properties are only assigned relative to other systems, and accordingly are nonlocal by their very nature. Further, due to the dynamical laws some quantum states (for example, the states characterizing the set up of Myrvold's theorem) may also involve a more radical type of nonlocality, namely e-nonlocality: The value of apparently local quantities may not be the same on two spacelike hypersurfaces that intersect the spacetime region in which the system they pertain to is located. Also, due to the probability assignment, properties that are related to different systems have no joint probabilities, and accordingly the presupposition that apparently local properties always have definite joint probabilities fails.

Our suggested relational modal interpretation also circumvents the other no-go theorems for relativistic modal interpretation. Since Myrvold's theorem is a generalization of Arntzenius's theorem, this interpretation similarly evades Arntzenius's theorem. And as we demonstrate in Berkovitz and $\mathrm{Hemmo}^{(23)}$, due to the holistic nature of the dynamics of properties (see Sec. 5.2), it also escapes Dickson and Clifton's no-go theorem. 
The relational modal interpretation does not only circumvent all the known no-go theorems for relativistic modal interpretation, it also offers an adequate solution to the measurement problem ${ }^{(23)}$ and it provides explanation for why certain properties of composite systems may fail to decompose into the properties of their subsystems, i.e. why the so-called 'property composition' and 'property decomposition' fail (see Sec. 4 and 5.1). Furthermore, due to the fact that the relational modal interpretation assigns properties in all (orthonormal) bases, it does not face the challenge of justifying the common (yet largely unmotivated) assumption of all the other modal interpretations that properties are only assigned in certain preferred (orthonormal) bases.

While the relational modal interpretation offers good prospects for solving the measurement problem and reconciling quantum mechanics with relativity, it may be objected that it is quite radical. We do not find this objection compelling. Indeed, the picture of physical reality portrayed by this interpretation is very different from the ones portrayed by the non-relational interpretations of quantum mechanics. But, in the history of physics the conception of physical reality has undergone a number of radical changes. 
In fact, orthodox quantum mechanics and its mainstream interpretations themselves mark a radical shift from classical physics.

We think that the merits of any interpretation of quantum mechanics have to be judged mainly on the basis of the interpretation's consistency, its empirical adequacy and its compatibility with other major theories. The relational modal interpretation seems to fare well on all these accounts. As far as we can see, it is consistent, it is empirically adequate and it provides good reasons to believe that quantum mechanics could be reconciled with relativity theory. We believe that the main challenge for the relational modal interpretation is the challenge from multiplicity. As we have seen in Sec. 6, there are various subsets of relational brain properties that could account for our beliefs about the physical systems that appear in our experience, and the question is which of these brain properties actually account for them. We believe that this question is not unique to the relational modal interpretation, yet due to the proliferation of (unrelated) definite properties it appears to be more acute in the context of this interpretation. 


\section{ACKNOWLEDGEMENTS}

During our Ph.D. studies at Cambridge and in the years to follow, we frequently met with Jim Cushing, attended his presentations and discussed with him the philosophy of quantum mechanics. Jim's intellectual curiosity and honesty, his enthusiasm and commitment to clarifying the foundations of quantum mechanics, his wealth of knowledge, and his gentle and generous nature gave us a source of inspiration. We would like to thank the editors, Michael Dickson, Sebastiano Sonego and Antony Valentini for the invitation to contribute to this volume. For discussions and comments on earlier versions of this paper, we are grateful to Guido Bacciagaluppi, Jeremy Butterfield, Richard Healey, Michael Redhead, Rafael Sorkin, Rob Spekkens and especially Wayne Myrvold, Itamar Pitowsky and Antony Valentini. We are also grateful to audiences at the Philosophy of Physics Discussion Group, Sub-faculty of Philosophy, Oxford University; Foundations of Science Colloquium, Utrecht University; the Philosophy Department, Freiburg University; the Committee for Philosophy and the Sciences, University of Maryland College Park; and the Perimeter Institute for Theoretical Physics, Waterloo, Canada. For financial support, JB would like to thank the Faculty of Arts 
and Sciences, the University of Maryland Baltimore County, and the Philosophy, Probability and Modelling Group, Center for Junior Researchers, Konstanz University. JB would also like to thank the Centre for Philosophy of Natural and Social Sciences, London School of Economics, and the Philosophy, Probability and Modelling Group for providing him with stimulating environments during the development of this paper.

\section{REFERENCES}

1. D. Dieks and P. Vermaas (eds.), The Modal Interpretation of Quantum Mechanics (Kluwer, Dordrecht, 1998).

2. M. Dickson, "Modal interpretations of quantum mechanics," in The Stanford Encyclopedia of Philosophy (Winter 2002 Edition), Edward N. Zalta, ed., http://plato.stanford.edu/archives/win2002/entries/qm$\operatorname{modal} /(2002)$.

3. G. Bacciagaluppi, "Delocalized properties in the modal interpretation of a continuous model of decoherence," Found. Phys. 30, 1431-1444 (2000). 
4. M. Dickson and R. Clifton, "Lorentz invariance in modal interpretations," in Ref. 1, pp. 9-47.

5. F. Arntzenius, "Curiouser and curiouser: problems for modal interpretations of quantum mechanics," in Ref. 1, pp. 337-377.

6. W. Myrvold, "Modal interpretations and relativity," Found. Phys. 32, 1773-1784 (2002).

7. S. Kochen "The interpretation of quantum mechanics," unpublished notes (1979).

8. G. Bene and D. Dieks, "A perspectival version of the modal interpretation of quantum mechanics and the origin of macroscopic behavior," Found. Phys. 32, 645-671 (2002).

9. H. Everett, "Relative state' formulation of quantum mechanics," Rev. Mod. Phys. 29, 454-462 (1957).

10. C. Rovelli, "Relational quantum mechanics," Int. J. Theo. Phys. 35, 1637-1678 (1996).

11. C. Rovelli, "Half way through the woods," in The Cosmos of Science, J. Earman and J. D. Norton, eds. (University of Pittsburgh Press, 
Pittsburgh, 1997), pp. 180-223.

12. S. Saunders, "Relativism," in Perspectives on Quantum Reality, R. Clifton, ed. (Kluwer, Dordrecht, 1996), pp. 125-142.

13. S. Saunders, "Time, quantum mechanics and probability," Synthese 114, 373-404 (1998).

14. F. Laudisa and C. Rovelli, "Relational quantum mechanics," The Stanford Encyclopedia of Philosophy (Spring 2002 Edition), Edward N. Zalta, ed., http://plato.stanford.edu/archives/spr2002/entries/qm-rel ational/(2002).

15. S. Kochen, "A new interpretation of quantum mechanics," in Symposium on the Foundations of Modern Physics, P. Lahti and P. Mittelstaedt, eds. (World Scientific, Singapore, 1985), pp. 151-169.

16. D. Dieks, "Resolution of the measurement problem through decoherence of the quantum state," Phys. Lett. A 142, 439-446.

17. R. Healey, The Philosophy of Quantum Mechanics: An Interactive Interpretation (Cambridge University Press, Cambridge, 1989).

18. P. Vermaas and D. Dieks, "The modal interpretation of quantum me- 
chanics and its generalization to density operators," Found. Phys. 25, 145-158 (1995).

19. J. Bub, "Quantum mechanics without the projection postulate," Found. Phys. 22, 737-754 (1992).

20. D. Bohm, "A suggested interpretation of the quantum theory in terms of 'hidden variables': Parts I and II," Phys. Rev. 85, 166-179 and 180-193 (1952).

21. G. Bacciagaluppi and M. Dickson, "Dynamics for modal interpretations," Found. Phys. 29, 1165-1201.

22. P. E. Vermaas, "The pros and cons of the Kochen-Dieks and the atomic modal interpretation," in Ref. 1, pp. 103-148.

23. J. Berkovitz and M. Hemmo, "A relational modal interpretation of quantum mechanics," in preparation.

24. S. Kochen and E. Specker, "On the problem of hidden variables in quantum mechanics," J. Math. Mech. 17, 59-87 (1967).

25. A. Shimony, "The degree of entanglement," Ann. NY Acad. Sci. 755, $675(1995)$. 
26. A. Abouraddy, B. Saleh and A. Sergienko, "Degree of entanglement for two qubits," Phys. Rev. A 64, 050101(R) (2001).

27. Y. Aharonov and D. Albert, "Can we make sense out of the measurement process in relativistic quantum mechanics," Phys. Rev. D 24, 359-370 (1981).

28. G. N. Fleming and H. Bennett, "Hyperplane dependence in relativistic quantum mechanics," Found. Phys. 19, 231-267 (1989).

29. G. N. Fleming, "Lorentz invariant state reduction, and localization," in PSA 1988, Vol. 2, A. Fine and M. Forbes, eds. (Philosophy of Science Association, East Lansing, MI, 1989), pp. 112-126.

30. G. N. Fleming, "Just how radical is hyperplane dependence?," in Perspectives on Quantum Reality, R. Clifton, ed. (Kluwer, Dordrecht, 1995), pp. 11-28.

31. T. Maudlin, Quantum Non-Locality and Relativity (Blackwell, Oxford, 1994).

32. T. Maudlin, "Spacetime in the quantum world," in Bohmian Mechanics and Quantum Theory: An Appraisal, J. Cushing, A. Fine and S. Goldstein, eds. (Kluwer, Dordrecht, 1996), pp. 285-307. 
33. G. C. Ghirardi, A. Rimini and T. Weber, "Unified dynamics for microscopic and macroscopic systems," Phys. Rev. D 34, 470-479 (1986).

34. P. Pearle, "Combining stochastic dynamical state-vector reduction with spontaneous localisation," Phys. Rev. A 39, 2277-2289 (1989). 


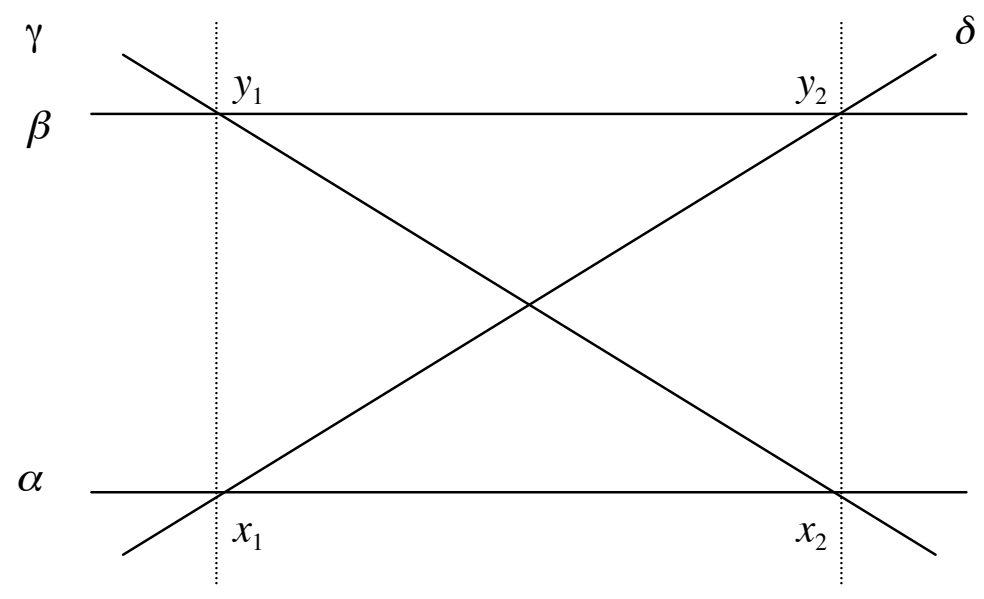

Fig. 1: The spacelike hypersurfaces used in Myrvold's theorem. 